\title{
Immunosuppressive therapy related adherence, beliefs and self-management in kidney transplant outpatients
}

This article was published in the following Dove Press journal:

Patient Preference and Adherence

\author{
Barbora Vankova' \\ Katerina Mala-Ladova' \\ Ales Antonin Kubena' \\ Josef Maly' \\ Sylvie Dusilova Sulkova ${ }^{2}$ \\ 'Department of Social and Clinical \\ Pharmacy, Faculty of Pharmacy in \\ Hradec Králové, Charles University, \\ Hradec Králové, Czech Republic; \\ ${ }^{2}$ Hemodialysis Centre, University \\ Hospital, Hradec Králové, Czech \\ Republic
}

Purpose: Kidney transplant (KTx) recipients should strictly adhere to their lifelong complex therapeutic regimen, and any barriers to medication adherence can weaken correct patient behavior. This study aimed to determine the adherence to immunosuppressive therapy (IS) in KTx adult outpatients in the Czech Republic, and attempted to gain a greater insight into their attitudes toward IS and self-management tasks.

Materials and methods: Pharmacist-led structured interviews were conducted to assess selfreported adherence to IS using the Czech version of the Medication Adherence Report Scale, in the context of attitudes toward IS in terms of necessity and concern scale of the Beliefs about Medicines Questionnaire. A specific questionnaire was developed to target IS self-management tasks. Medication records were also reviewed for IS serum levels, reflecting direct adherence measurement. Descriptive statistics were used to calculate adherence and self-management variables, and were analyzed by univariate and multivariate correlations, including the decision-tree method.

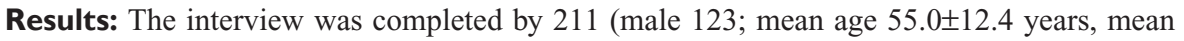
time $6.6 \pm 5.9$ years after KTx) of the total of 235 patients. Full adherence to IS was reported by $173(82.0 \%)$ patients. Most of them had IS serum levels within the therapeutic range, however, cyclosporine was associated with the highest variability $(P<0.001)$. Non-adherence and concerns increased over time after $\mathrm{KTx}(P<0.05)$. Despite the more common unintentional nonadherence $(P<0.001)$, relatively high concerns signified the risk of not taking IS as prescribed. Concerns also correlated with the perception of impaired health status $(P<0.01)$, as well as the occurrence of IS-related adverse effects $(P<0.001)$. The patients' awareness of their therapy was insufficient, and main gaps in self-management comprised inadequate sun protection, incorrect administration of IS, and unfamiliarity with the IS name, or their indications.

Conclusion: Although self-reported adherence to IS therapy was satisfactory, the comprehensive evaluation enabled the detection of greater concerns about IS, as well as underestimated self-management tasks that posttransplant interventions should target in the future.

Keywords: medication adherence, self-management, kidney transplantation, immunosuppression, beliefs about medicines

\section{Introduction}

Kidney transplantation (KTx) is the best treatment option for most patients with end-stage renal disease associated with longer survival and improved quality of life. However, lifelong immunosuppressive treatment (IS) is required to minimize the risk of graft rejection. Therefore, KTx recipients need to adhere to complex therapeutic regimens, including a combination of IS, along with other medications to prevent or treat comorbidities. ${ }^{1}$
Department of Social and Clinical Pharmacy, Faculty of Pharmacy in Hradec Králové, Charles University, Akademika Heyrovského I203, 50005 Hradec Králové, Czech Republic Tel +420 49506725 I Email malyj@faf.cuni.cz 
In general, medication adherence is the key to therapeutic success. According to the WHO, it can be defined as the extent to which a person's behavior corresponds with the agreed recommendations from their health care professionals. ${ }^{2}$ The term adherence goes beyond simply taking medication, because it reflects the broad-range of health-related behavior, including dietary habits, or lifestyle. ${ }^{3}$ Medication non-adherence, therefore, brings about both personal and economic costs associated with increased morbidity, hospitalization, and mortality. ${ }^{4}$ Non-adherence to posttransplant immunosuppression (IMNA) represents one of the leading preventable causes of graft rejection, which may eventually end in failure of graft functioning. Among non-adherent groups, the risk of graft rejection is increased by seven-fold. ${ }^{5}$ Despite its serious consequences, IMNA remains a common problem in KTx outpatients, with occurrence ranging between $22 \%$ and $68 \%$ depending on measurement modalities and sampling methods. ${ }^{1}$

The established current approach suggests that medication adherence should not be considered as a dichotomous variable (adherent vs non-adherent), ${ }^{6}$ yet should be considered as a dynamic process influenced by a variety of factors, which may even change over time. The absence of a gold standard in measurement also limits objective comparison among different studies, however, the combination of subjective and objective methods is regarded as the most accurate. ${ }^{7}$ In this context, an important distinction should also be drawn between unintentional and intentional non-adherence. Unintentional non-adherence occurs when patients cannot take their medication as prescribed due to factors beyond their control (eg, forgetfulness, impaired ability to understand instructions, etc.). Intentional non-adherence occurs when patients decide not to take their medication, usually based either on personal beliefs, or due to negative attitudes toward the treatment. According to the Necessity-Concerns Framework, the patients' motivation to adhere is influenced by their personal needs for medication (necessity beliefs), as well as by their concerns regarding potential adverse effects. ${ }^{8}$ This type of belief regarding the treatment has been shown to be an independent risk factor of non-adherence. ${ }^{9}$

As widely accepted, KTx is both stressful and a lifechanging milestone. Patients must quickly adopt to an altogether different lifestyle, as well as to medicines with a certain diet to minimize the risks of long-term IS. While pursuing a complex therapeutic regimen, they must be strictly adherent as far as possible and persist with it during the whole posttransplant period. Hence, the purpose of our study was to evaluate the situation among KTx outpatients in the Czech
Republic. We aimed to determine patients' beliefs about their treatment and self-reported adherence to IS, as well as the overall posttransplant self-management behavior in relation to sociodemographic, clinical, and time variables. For us, these data will be useful for detecting patients with poor medication-taking behavior, while the multidimensional view of the study may further allow the development of targeted interventions whenever needed.

\section{Materials and methods Study cohort}

The cross-sectional study was carried out in a 12-month period, from March 2016 to March 2017, at the Hemodialysis Center, University Hospital Hradec Králové, in the Czech Republic. Patients $\geq 18$ years of age and at least 3 weeks after KTx were invited during their scheduled follow-up with the nephrologist. Patients had to sign the informed consent. The exclusion criteria were initial or antirejective IS, cognitive disorders, hospitalization, or disapproval to being enrolled. The study design was pre-approved by the Ethics Committee of the University Hospital Hradec Králové and conforms to the provisions of the Declaration of Helsinki.

\section{Data collection and measurement}

All data were obtained through a uniform pharmacist-led structured interview (BV), supplemented with a review of medical documentation. Each interview took 20-30 minutes. At the beginning, patients were acquainted with the study, and subsequently asked for the name of all the prescribed IS medications. Sociodemographic characteristics, including education, occupational status, hometown, and household income, were completed. Patients were also asked to rate their health status using the 5-point Likert scale. Self-reported medication adherence was measured by the Czech version of the Medication Adherence Report Scale (MARS-CZ), which was validated in an interview. ${ }^{10}$ The MARS-CZ can be employed in various diseases with similar dosing regimen, as the items are focused on the patients' intentional or unintentional changes in prescribed dosages. The same benefits apply to the Beliefs about Medicines Questionnaire (Czech version BMQ-CZ), wherein the beliefs about IS were assessed using its specific subscale. ${ }^{11}$ Cronbach alphas indicated good internal reliability (for MARS-CZ $\alpha=0.67$, BMQ-CZ necessity $\alpha=0.75$, and BMQ-CZ concern $\alpha=0.73$ ). Both the MARS and the BMQ questionnaires were originally published by Horne et al. ${ }^{12,13}$

The MARS-CZ consists of five statements related to nonadherent behavior, of which three items reflect intentional 
non-adherence (eg, decision to miss a dose), while two reveal unintentional non-adherence (eg, forgetting to take). Each item is scored on 5-point Likert scale, with one indicating "always" and five indicating "never". The maximum of 25 points represents full adherence. The continuous score (0-25 points) was combined with dichotomous variables using the cut-point of 23 points to determine high ( $\geq 23$ points) and low ( $<23$ points) level of adherence. This cut-point was preferred in studies requiring the patients' strict medication adherence, established in agreement with the Czech validation study. ${ }^{10}$ The BMQ-CZ consists of 11 statements concerning personal beliefs about the necessity of the medication, as well as their concerns regarding potential adverse effects. The respondents' agreements with each statement were scored from strongly disagree (one point) to strongly agree (five points). The average score was calculated for each necessity and concern subscales.

The interviews were followed up by questions about the real use of IS, including self-management issues, as well as dietary and lifestyle habits. The questions were formulated based both on the reviewed literature and on the report received from the expert panel, which was composed of the responsible nephrologist, nurse and clinical pharmacist. A pilot study was conducted to test the readability of the questionnaire. From the area of self-management, patients' self-monitoring of blood pressure, their approach to sun protection, preventive examination (eg, vaccination), food avoidance, salt restriction, as well as their smoking status were determined. Questions focusing on IS pharmacotherapy were divided into a general part and a section specific to each IS substance. The general part included daily drug preparation, drug use aids, use of over-the-counter, and dietary supplements. The specific section referred to the use of IS in relation to food and dosage regimen, liquids for swallowing the pill, satisfaction with the drug form, and the subjective feeling of any adverse effects.

Simultaneously, medication records were reviewed during each interview, especially to discuss the complete pharmacotherapy and also the serum concentrations of the main IS, including tacrolimus (TAC), cyclosporine (CYSP), sirolimus (SIR), and everolimus (EVE). Serum levels of these ISs were regularly monitored in the hospital. Based on hospital guidelines, the target range for TAC was $10-15 \mu \mathrm{g} / \mathrm{L}$ in newly transplanted, and $5-10 \mu \mathrm{g} / \mathrm{L}$ in patients at least 3 months after KTx. For CYSP, the limit was 80-120 nmol/L, whereas 4-9 $\mu \mathrm{g} / \mathrm{L}$ for SIR and EVE. The cut-off values were adapted whenever the medical record indicated individual treatment. Before measurement, patients were instructed not to take their morning dose, but after the sampling instead. Targeting the IS serum concentration, the limits were assessed for all ISs ranging from 0 to 1 . Negative values stood for sub-therapeutic concentrations that may indicate poor medication adherence. The risk of low concentrations associated with possible drug interactions, was also determined. Counting with long-term individual dose adjustments, it was not necessary to specify pharmacogenomics. In addition, further clinical characteristics were collected from the patients' medical records, that is, diagnosis, family history of kidney disease, causes of renal failure, time interval after KTx, parameters indicating kidney functioning, as well as other data otherwise not covered by the interviews.

\section{Statistical analysis}

Data were analyzed using Wolfram, Mathematica, version 11.2. Methods of descriptive statistics were used for characteristics of the tested cohort by the mean of frequencies for nominal and mean, median $\pm \mathrm{SD}$ for metric items. Due to the frequent ordinal nature of data derived mainly from questionnaires, the use of non-parametric tests was preferred. Correlations between pairs of numerical or ordinal variables were measured by Kendall's tau $(\tau)$. The signs and magnitude of the differences in position were tested by the Wilcoxon signed-rank test for two levels of independent factors and the Kruskall-Wallis test for multiple levels of independent factors. The $P$-value of $<0.05$ was considered as statistically significant.

In addition to the statistical significance value, the corresponding effect size was calculated. However, selected coefficients were used for the effect size, and in the case of correlations, additional tests had to be performed: $\mathrm{d}$ for two groups and $\eta 2$ for multiple group differences in position, Pearson $r$ for correlation of two variables and $w$ for difference in frequencies of individual values of nominal variables. Only medium or large effect sizes according to Cohen's convention are mentioned in the results. ${ }^{14}$

The difference of IS dispersion was tested by the Conover squared ranks test. Lower level of between-subject variability was corresponded with the more successful control of obtained IS serum level. The frequency of variability, potentially leading to sub-therapeutic IS concentrations, was compared between the groups by chi-squared test.

The dependence of a numeric variable (IS serum level or the MARS-CZ) on more non-independent binary variables (recommendations in the area of self-management) was analyzed using Chi-square automatic interaction detection (CHAID) decision-tree method. 


\section{Results}

\section{Participants' characteristics}

During the study period, there were 412 KTx outpatients in posttransplant care at the Hemodialysis Center, of whom 235 patients were invited to participate. The interviews were conducted with 211 patients (89.9\%), as 24 (10.1\%) declined, due to lack of time or interest. The study cohort included 123 (58.3\%) male patients. The mean age was $55.0 \pm 12.40$ years. Approximately one-third of the patients were active workers, one-third were receiving disability pension, and the remaining one-third receiving retirement pension.

The mean time after KTx was $6.6 \pm 5.89$ years with chronic glomerulonephritis as the most frequent cause of renal failure. Patients used on average 11 drugs per day. The most commonly prescribed IS regimen was a combination of TAC, mycophenolate, and prednisone (PRE). In total, $174(82.5 \%)$ respondents could name their main IS, but only 74 (35.1\%) patients named all their prescribed ISs. Complete sociodemographic, KTx, and treatment characteristics are shown in Table 1.

\section{Self-reported adherence to immunosuppressives}

Self-reported adherence to IS measured by the MARS-CZ was high (mean $24.7 \pm 0.95$, median 25 , min 16 , and max 25 ). Unintentional IMNA was significantly more common than intentional IMNA ( $\mathrm{d}=0.33, P<0.001)$. The score of the MARS-CZ depended on the specific main IS $\left(\eta^{2}=0.02\right.$, $P=0.040$ ). For instance, all patients using SIR were fully adherent. However, this correlation may be biased due to the ceiling effect, as most patients gained the maximum score at the MARS-CZ. Generally, less than perfect adherence $(<25$ points) was found in $38(18.0 \%)$ cases and low adherence $(<23$ points) in $6(2.8 \%)$ cases. Significant correlation was found between self-reported adherence and higher age at the time of KTx ( $\tau=0.03, r=0.20, P<0.001)$, whereas the score of the MARS-CZ decreased with time after KTx ( $\tau=-0.12, r=-0.13, P=0.026$ ). Correlations between the MARS-CZ and relevant sociodemographic and clinical variables are summarized in Table 2.

\section{Perceptions and beliefs about immunosuppressives}

The mean necessity and concern score of the BMQ-CZ were $4.7 \pm 0.5$ and $2.3 \pm 1.0$, respectively. Only small deviations among necessity responses presumed the presence of a ceiling effect. Patients agreed the most with the statement
Table I Sociodemographic, clinical, and medicine characteristics $(n=2 \mid$ I)

\begin{tabular}{|c|c|}
\hline \multicolumn{2}{|l|}{ Characteristics } \\
\hline \multicolumn{2}{|l|}{ Gender, n (\%) } \\
\hline Male (\%) & $123(58.3)$ \\
\hline \multicolumn{2}{|l|}{ Age (years) } \\
\hline Median (min, max) & $57.0(25-77)$ \\
\hline \multicolumn{2}{|l|}{ Education, n (\%) } \\
\hline Secondary school & $22(10.4)$ \\
\hline High school & $172(8 \mid .5)$ \\
\hline Post/undergraduate & $17(8.1)$ \\
\hline \multicolumn{2}{|l|}{ Occupation, n (\%) $)^{\mathrm{a}}$} \\
\hline Active worker & $65(29.4)$ \\
\hline Disability pension & $76(34.4)$ \\
\hline Retirement pension & $77(34.8)$ \\
\hline \multicolumn{2}{|l|}{ Hometown (inhabitants), n (\%) } \\
\hline$<10,000$ & $143(67.8)$ \\
\hline \multicolumn{2}{|l|}{ Living alone, $\mathrm{n}(\%)$} \\
\hline Yes & $43(20.4)$ \\
\hline \multicolumn{2}{|c|}{ Income per month (Czech crown), n (\%) } \\
\hline$<30,000$ & $140(66.3)$ \\
\hline \multicolumn{2}{|l|}{ Satisfaction with the income, $\mathrm{n}(\%)$} \\
\hline Yes & $12 \mid(57.3)$ \\
\hline \multicolumn{2}{|l|}{ Time after KTx (months), n (\%) } \\
\hline$\leq 3$ & $16(7.6)$ \\
\hline $4-12$ & $21(10.0)$ \\
\hline $13-24$ & $25(11.8)$ \\
\hline$>24$ & $149(70.6)$ \\
\hline \multicolumn{2}{|l|}{ Repeated transplants, n (\%) } \\
\hline Yes & $23(10.9)$ \\
\hline \multicolumn{2}{|l|}{ Type of donor, n (\%) } \\
\hline Cadaveric & $200(94.8)$ \\
\hline Living & II (5.2) \\
\hline \multicolumn{2}{|l|}{ Cause of renal failure, $n(\%)$} \\
\hline Chronic glomerulonephritis & $51(24.2)$ \\
\hline IgA nephropathy & $32(15.2)$ \\
\hline Renal polycystosis & $38(18.0)$ \\
\hline \multicolumn{2}{|c|}{ Family history of kidney disease, $\mathrm{n}(\%)$} \\
\hline Positive & $55(26.1)$ \\
\hline Not available & $22(10.4)$ \\
\hline \multicolumn{2}{|c|}{ Estimated glomerular filtration rate $(\mathrm{mL} / \mathrm{s}), \mathrm{n}(\%)^{\mathrm{c}}$} \\
\hline$>1.5$ & $4(1.9)$ \\
\hline $1-1.5$ & $45(21.3)$ \\
\hline $0.5-1$ & $130(61.6)$ \\
\hline$<0.5$ & $32(15.2)$ \\
\hline \multicolumn{2}{|l|}{ Medication, mean (SD) } \\
\hline Prescription & $11.13 \pm 2.90$ \\
\hline Over the counter & $0.86 \pm 1.09$ \\
\hline
\end{tabular}

(Continued) 
Table I (Continued)

\begin{tabular}{|l|l|}
\hline \multicolumn{2}{|l|}{ Characteristics } \\
\hline \multicolumn{2}{|l|}{ Immunosuppression, $\mathrm{n}(\%)^{\mathrm{a}}$} \\
\hline Tacrolimus & $125(59.2)$ \\
\hline Cyclosporine & $59(28.0)$ \\
\hline Sirolimus & $30(13.3)$ \\
\hline Everolimus & $2(0.9)$ \\
\hline Mycophenolate & $169(80.1)$ \\
\hline Prednisone & $201(95.3)$ \\
\hline Azathioprine & $7(3.3)$ \\
\hline Knowledge of the IS name, $\mathrm{n}(\%)^{\mathrm{a}}$ & \\
\hline Tacrolimus & $105(84.0)$ \\
\hline Cyclosporine & $47(79.7)$ \\
\hline Sirolimus/everolimus & $24(80.0)$ \\
\hline Mycophenolate & $117(69.2)$ \\
\hline Prednisone & $77(38.3)$ \\
\hline Azathioprine & $6(85.7)$ \\
\hline Perception of health condition, $\mathrm{n}(\%)$ & \\
\hline Excellent & $16(7.6)$ \\
\hline Very good & $35(16.6)$ \\
\hline Good & $113(53.6)$ \\
\hline Satisfying & $41(19.3)$ \\
\hline Bad & $6(2.9)$ \\
\hline
\end{tabular}

Notes: aMultiple answers possible; bexchange rate on 12/04/20 I8: one euro $=25.3$ Czech crown; 'eGFR using CKD-EPI.

Abbreviations: IS, immunosuppression; KTx, kidney transplantation.

that medicines protect their health status from turning worse (96.2\% agreed or strongly agreed), and that without the medicines, they would be very ill (94.8\%). As previously with the MARS-CZ, the necessity of the treatment significantly correlated with higher age at the time of $\operatorname{KTx}(\tau=0.19, r=0.20$, $P<0.001)$, whereas concerns increased significantly with time after KTx $(\tau=0.10, r=0.15, P=0.035)$ (Table 2$)$. Patients expressed their concerns most frequently about the adverse effects of long-term IS therapy (46.5\%). The manifestation of adverse effects caused by IS was admitted by 102 (48.3\%) respondents, but only 70 discussed it with their nephrologists. Furthermore, one-third of the patients (32.7\%) were worried that they had to take IS at all. The concern scale correlated with the occurrence of adverse effects $(\mathrm{d}=0.57, P<0.001$, medium effect size), as well with a worsening perception of their health status ( $\tau=-0.17, r=-0.22, P=0.002$ ). As presented in Table 1, most respondents considered their health condition as "good", whereas $6(2.9 \%)$ patients perceived it as "bad". This perception altered with increasing age ( $\tau=-0.25$, $r=-0.30, P<0.001$, medium).

\section{Actual use of IS and self-management}

The majority of respondents used IS after meals. TAC on an empty stomach was taken by $43(34.4 \%)$ respondents. The IS dosage form was scored as satisfactory for the patients; nevertheless, the pill size was mentioned as a problem for 29 patients (mycophenolate 10; TAC 9; CYSP 7; and PRE 3). Another 10 respondents complained about the bitter taste of PRE, while one respondent was not satisfied with the smell of the CYSP capsule. Better adherence scores correlated with greater willingness to use sun protection $(P=0.004$, CHAID). However, the awareness of sun protection, as well as regular use of sunblock cream were rather low. Compared with data on the general population, a smaller number of patients were

Table 2 Kendall's correlations of self-reported adherence, beliefs, and IS serum levels with relevant sociodemographic and clinical characteristics $(\mathrm{n}=2 \mathrm{I} \mathrm{I})$

\begin{tabular}{|l|l|l|l|l|}
\hline Characteristics & MARS-CZ & $\begin{array}{l}\text { Relative IS } \\
\text { serum level' }\end{array}$ & $\begin{array}{l}\text { BMQ-CZ, } \\
\text { necessity }\end{array}$ & $\begin{array}{l}\text { BMQ-CZ, } \\
\text { concern }\end{array}$ \\
\hline Age (years) & $0.179^{* *}$ & 0.001 & $0.14 I^{* *}$ & -0.0003 \\
\hline Age at KTx (years) & $0.208^{* * *}$ & -0.009 & $0.189^{* * *}$ & -0.057 \\
\hline Time after KTx (months) & $-0.124^{*}$ & 0.020 & -0.090 & $0.10 I^{*}$ \\
\hline MARS, total & & 0.017 & $0.165^{* *}$ & $-0.118^{*}$ \\
\hline Relative IS serum level' & 0.017 & & 0.022 & 0.029 \\
\hline BMQ, necessity & $0.165^{* *}$ & 0.022 & -0.059 \\
\hline BMQ, concern & $-0.118^{*}$ & 0.029 & -0.059 & \\
\hline Estimated glomerular filtration rate $(\mathrm{mL} / \mathrm{s})^{2}$ & 0.070 & 0.073 & 0.024 & 0.031 \\
\hline Number of medication per day & 0.073 & 0.020 & $0.133^{*}$ & 0.086 \\
\hline Educational status & 0.014 & -0.082 & $-0.248^{* * *}$ & 0.055 \\
\hline Perception of health status & 0.098 & 0.031 & -0.099 & $-0.166^{* *}$ \\
\hline
\end{tabular}

Notes: 'Target range for tacrolimus was $10-5 \mu \mathrm{g} / \mathrm{L}$ in newly transplanted and 5-10 $\mu \mathrm{g} / \mathrm{L}$ in others; $80-120 \mathrm{nmol} / \mathrm{L}$ for cyclosporine and 4-9 $\mu \mathrm{g} / \mathrm{L}$ for sirolimus; ${ }^{2} \mathrm{eGFR}$ using CKD-EPI. $* P<0.05 ; * * P<0.01 ; * * * P<0.001$.

Abbreviations: BMQ-CZ, Beliefs about Medicines Questionnaire - Czech version; IS, immunosuppression; KTx, kidney transplantation; MARS-CZ, Medication Adherence Report Scale - Czech version. 
active smokers. Respondents with stronger beliefs about the need of IS were also more aware of the potential drug-food interaction with CYP450 inhibitors, for example, the need of avoiding grapefruit, pomelo, and pomegranate consumption ( $\tau=0.13, r=0.15, P=0.037$ ). Multivariate correlations did not detect other statistically relevant associations. Complete selfmanagement tasks are summarized in Table 3.

\section{IS serum levels}

The comparison of IS serum levels among the main IS (EVE excluded due to low number of recipients) is presented in Figure 1. Most patients presented IS serum levels within the therapeutic range ( $0-1)$ with median $0.40,0.30$, and 0.34 of CYSP, SIR, and TAC, respectively. IS differed in the IQR, where CYSP was associated with the highest statistical variability in the serum level $(P<0.001$, Conover test). Furthermore, the use of CYSP was at the greatest risk of reaching the serum level below the lower limit of the therapeutic range (w $=0.59, P=0.035$, large). No correlation was found with the MARS-CZ and the BMQ-CZ (Table 2), however, low serum levels of CYSP, SIR, and TAC were found in 14 (23.7\%), $1(3.8 \%)$, and $14(11.2 \%)$ patients, respectively. Only a limited number of patients received SIR, but the results showed relatively high number of cases with serum levels beyond the upper limit of the therapeutic range. Due to this insight, TAC seemed to be the preferred IS, ensuring the most stable immunosuppression.

\section{Discussion}

Adherence to IS was observed as satisfactory, however, with deteriorating tendencies over time after KTx. Furthermore, younger age at the time of KTx was closely related to decreased adherence and beliefs. Although unintentional IMNA was more common, relatively high concerns about long-term IS therapy signified the risk of intentional IMNA.

Current research has shown the need of strict adherence to IS during the whole posttransplant period. In KTx outpatients, IMNA is considered as the cause of about half of all rejection episodes, as well as $15 \%$ of graft loss..$^{15}$ The actual situation in the Czech Republic remains unknown. A Slovakian study on 252 KTx patients reported 37 (14.7\%) cases of IMNA, when the indications of non-adherence were the caregivers or family members suspicion, long-term variability of IS serum levels, as well as avoidance of follow-up visits. In this study, the leading causes of IMNA were forgetting to take IS and the fear of undesirable effects of IS. All non-adherent patients showed positivity for minimum one donor-specific
Table 3 Self-management tasks $(n=2 \mid \mathrm{I})$

\begin{tabular}{|c|c|}
\hline \multicolumn{2}{|l|}{ Drug preparation and use } \\
\hline \multicolumn{2}{|l|}{ Taking pill with relation to food, $\mathrm{n}(\%)$} \\
\hline Tacrolimus on an empty stomach & $43(34.4)$ \\
\hline Prednisone after breakfast & $160(79.6)$ \\
\hline \multicolumn{2}{|l|}{ Liquid for swallowing the pill, $n$ (\%) } \\
\hline Only water or fruit tea & $138(65.4)$ \\
\hline Green or black tea, milk, coffee, etc & $73(34.6)$ \\
\hline \multicolumn{2}{|l|}{ Daily drug preparation, n (\%) } \\
\hline Himself/herself & $192(91.0)$ \\
\hline Family member & $20(9.5)$ \\
\hline Nurse & $\mathrm{I}(0.5)$ \\
\hline \multicolumn{2}{|l|}{ Use of drug aids, n (\%) } \\
\hline Pillbox & $114(54.0)$ \\
\hline Medication list & $119(56.4)$ \\
\hline None & $63(29.9)$ \\
\hline \multicolumn{2}{|l|}{ Dietary habits, n (\%) } \\
\hline Food avoidance ${ }^{1}$ & $92(43.6)$ \\
\hline Grapefruit, pomelo, and pomegranate consumption & $68(32.2)$ \\
\hline Salt restriction & $147(69.7)$ \\
\hline \multicolumn{2}{|l|}{ Lifestyle habits } \\
\hline \multicolumn{2}{|l|}{ Sun protection, n (\%) } \\
\hline Sunblock cream & $55(26.1)$ \\
\hline Proper clothes (eg, hat) & $73(34.6)$ \\
\hline Avoiding sunshine (at noon) & $110(52.4)$ \\
\hline Avoiding solarium & $211(100.0)$ \\
\hline Nothing & $58(27.5)$ \\
\hline \multicolumn{2}{|l|}{ Smoking, n (\%) } \\
\hline Current smoker & 24 (II.4) \\
\hline Ex-smoker & $58(27.5)$ \\
\hline Never smoked & $129(61.1)$ \\
\hline \multicolumn{2}{|l|}{ Monitoring of blood pressure, $\mathrm{n}(\%)$} \\
\hline Regularly & $114(54.0)$ \\
\hline When needed & $44(20.9)$ \\
\hline By a practitioner & $12(5.7)$ \\
\hline Week before medical appointment & $2(0.9)$ \\
\hline None & $39(18.5)$ \\
\hline Monitoring of body weight, n (\%) & $159(79.1)$ \\
\hline \multicolumn{2}{|l|}{ Preventive examination, $\mathrm{n}(\%)^{2}$} \\
\hline Influenza vaccine & $42(19.9)$ \\
\hline Pneumococcal vaccine & $5(2.4)$ \\
\hline Bone densitometry & $49(23.2)$ \\
\hline
\end{tabular}

Notes: 'Individual restriction of any kind of nourishment; ${ }^{2}$ Individual awareness of selected preventive examination.

antibody, whereas the graft function was worse in all patients with IMNA. ${ }^{16}$

The standardized questionnaires used in our study allowed the identification of patients with increased risk of IMNA. ${ }^{17}$ Due to their simplicity and low financial burden, 


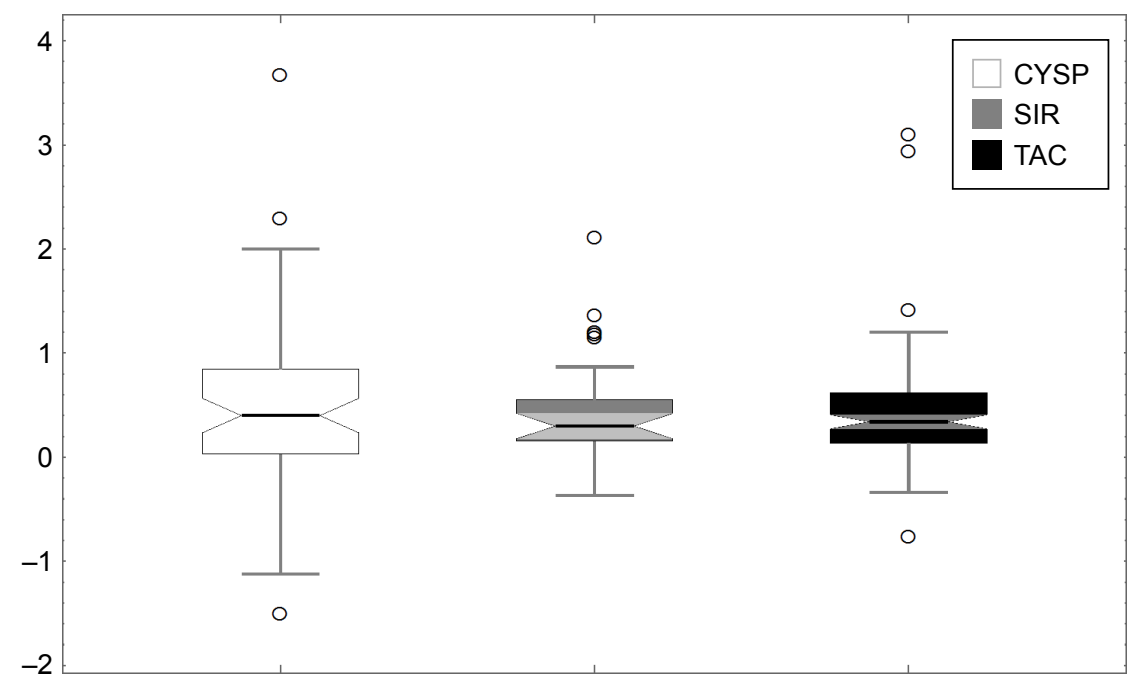

Figure I Serum levels of main immunosuppressives.

Abbreviations: CYSP, cyclosporine; SIR, sirolimus; TAC, tacrolimus.

an affordable method was provided offering comparable results across different health conditions. On the other hand, there is always certain risk posed by the respondents' overvaluation, either due to fears of disappointing their health care providers, or simply because of different perceptions toward medication-related behavior. The so-called social desirability bias might also have occurred in our study, especially in cases when the questionnaires were delivered in face-to-face interviews. Furthermore, data from different types of questionnaires may not be easily comparable with each other. We found two cross-sectional studies using the MARS questionnaire to estimate adherence to IS in patients after KTx, with slightly different results. In these studies, about half of the respondents were fully adherent, while $<10 \%$ had $<23$ points. ${ }^{18,19}$ In our study, full adherence was reported by $>80 \%$ of all respondents, and only $2.8 \%$ of them had $<23$ points. Similar results were found in another study from the Czech Republic, in which the MARS-CZ was used to determine self-reported adherence to statins. ${ }^{10}$ Similar findings to ours were also observed with other scales (eg, the Basel Assessment of Adherence with Immunosuppressive Medication Scales), even if a less strict scoring method was used..$^{20,21}$ On the other hand, the results of the Immunosuppressant Therapy Adherence Scale varied from $60 \%{ }^{22}$ to $>90 \%{ }^{23}$ of fully adherent respondents. Consistent with our study, the effect sizes of most significant results were small, or were not reported at all. This may be another explanation for high heterogeneity in results among studies.

In cases when standardized questionnaires were combined with patient interviews, the accuracy of evaluating IMNA remained low, yet increased the possibility of identification of multiple risk factors of IMNA for each respondent. This is beneficial, as the etiology of IMNA is multifactorial. Younger age and pre-transplant medication non-adherence were considered as major risk factors. ${ }^{22,24}$ Similarly, younger patients showed decreased adherence in our study. This may be caused by their busy lifestyles or higher concerns about adverse effects of long-term immunosuppression (eg, cardiovascular disease and cancer) ${ }^{25,26}$ Non-adherence also increased with time after KTx. The same results were found in a study by De Geest et $\mathrm{al}^{27}$ and Massey et $\mathrm{al},{ }^{20}$ where IMNA increased significantly between 6 weeks and 6 months after KTx from $17 \%$ to $27 \%$. This can be explained as some recipients might have experienced burn-out syndrome, whereas others gained greater confidence in the viability of the kidney, or felt better. ${ }^{26}$

A high number of non-adherent KTx outpatients reported unintentional IMNA. Forgetfulness was reported as the most common reason. ${ }^{28}$ Generally, respondents considered IS very important for their health, but this attitude was observed to decrease over time. The result corresponded with data of all similar studies conducted on KTx patients ${ }^{18,19,29}$ as well as all studies conducted on chronically ill patients in the Czech Republic. ${ }^{10,30}$ However, few studies questioned this particular association between beliefs and adherence. ${ }^{31}$ In a study by Massey et al, patients after KTx reported a high level of perceived necessity and relatively few concerns about their IS, but IMNA still increased significantly over a period of 18 months. ${ }^{20}$ Higher concerns about IS therapy were related to the worsening perception of their health condition, as well as the occurrence of adverse effects. These concerns increased with time after KTx. Greater deficiencies were 
found in self-management, for example, the absence of any sun protection, as well as incorrect administration of TAC and PRE, were the most serious ones.

The data obtained from self-report correlated with the type of IS, although not with IS serum concentrations. Higher rates of sub-therapeutic level and higher statistical variability were related to CYSP therapy. Even if nonadherence cannot be determined by single measurement of IS serum concentration, the IMNA might be a major factor contributing to high IS serum level variability. Therefore, suspected IMNA still must be considered, even if the ceiling effect of the MARS-CZ was noticed. Moreover, the questionnaire referred to all prescribed ISs, whereas serum levels were measured only for the main IS. In a comparison, no association between self-reported adherence to IS and IS serum concentrations was found in the study by Griva et al, however, lower rates of IMNA based on IS serum levels than on self-reports were observed. ${ }^{19}$ Serum concentrations reflect only medication taken prior to visit, while the selfreported questionnaire estimates adherence patterns over a longer period and more generally. Concerning the variability of CYSP serum levels, the explanation could be the need for two daily doses as CYSP has a shorter biological half-life, or higher age among CYSP users due to the longer registration period on the market.

In our study, the patients' knowledge of their own IS therapy was often insufficient, which included not only being unfamiliar with the type of ISs and indications, but also incorrect drug administration in relation to food or daily schedule. Concerning self-management, inadequate sun protection unnecessarily increased the risk of skin cancer. Providing the additional interventions, such as educational materials and repeated motivational interviewing, could be effective in reducing these risk factors in KTx outpatients. ${ }^{32,33}$ In cases of unintentional non-adherence, patients may profit from establishing a daily medication routine, and also by simplifying the daily drug regimen with the use of pillboxes, reminders, or other technology-based devices. On the other hand, motivational interviewing may be helpful in patients intentionally refusing the suggested therapeutic plan. ${ }^{31}$ Essentially, each health care provider, according to their competencies, may further promote individually the patients' adherence to the therapy and self-management.

The limitation of our study was the unicentric crosssectional design with all the limits of engaged adherence measuring, as aforementioned, and with the impossibility to cover outcome surveillance, for example, the onset of rejection episode, or progression of graft dysfunction.
Similarly, our study did not reflect on the clinic-dependent posttransplant education either.

\section{Conclusion}

In conclusion, patients after KTx may be non-adherent to the therapy. Among all respondents, the medication adherence and beliefs related to the necessity of IS therapy were often satisfactory, however, worsened over the time passed after KTx. Few patients admitted forgetting, but relatively high concerns about long-term IS use signified the risk of intentional IMNA. Despite the intensive posttransplant care and frequent medical appointments, the patients' attitudes toward the treatment, their knowledge of IS, as well as adherence to self-management issues seemed to be underestimated. Self-reported questionnaires enabled detecting persons at higher risk of IMNA, yet also help the identification of individual barriers. This could be employed in the development of targeted interventions for patients with IMNA, as well as to propose some general enhancements in posttransplant care. Our results may also motivate health care professionals in implementation of new approaches.

\section{Acknowledgments}

This study was supported by Charles University (Project SVV 260 417, PROGRES Q40-14, and PROGRES Q42). We thank the University Hospital Hradec Králové for providing the clinical data for this research and the nurses (particularly Zuzana Sircekova) at the Hemodialysis Center for their contribution in addressing patients to participate in the study.

\section{Author contributions}

All five authors of this manuscript 1) made substantial contributions to conception and design, acquisition of data, or analysis and interpretation of data; 2) drafted the article or revised it critically for important intellectual content; 3) gave final approval for the version to be published; and 4) were in agreement to be held accountable for all aspects of the work, including ensuring that questions related to the accuracy or integrity of any part of the work are appropriately investigated and resolved.

\section{Disclosure}

The authors report no conflicts of interest in this work.

\section{References}

1. Neuberger JM, Bechstein WO, Kuypers DRJ, et al. Practical recommendations for long-term management of modifiable risks in kidney and liver transplant recipients. Transplantation. 2017;101(Suppl 2): S1-S56. 
2. Sabaté E. Adherence to Long-Term Therapies: Evidence for Action. Geneva, Switzerland: World Health Organization [WHO]; 2003.

3. Simpson SH, Eurich DT, Majumdar SR, et al. A meta-analysis of the association between adherence to drug therapy and mortality. BMJ. 2006;333(7557): 15 .

4. NICE 2009 Medicines adherence: Involving Patients in Decision About Prescribed Medicines and Supporting Adherence. London, UK: Royal College of General Practitioners; 2009.

5. Butler JA, Roderick P, Mullee M, Mason JC, Peveler RC. Frequency and impact of nonadherence to immunosuppressants after renal transplantation: a systematic review. Transplantation. 2004;77(5):769-776.

6. Vrijens B, Antoniou S, Burnier M, de la Sierra A, Volpe M. Current situation of medication adherence in hypertension. Front Pharmacol. 2017;8:100.

7. Lam WY, Fresco P. Medication adherence measures: an overview. Biomed Res Int. 2015;2015:217047.

8. Horne R, Chapman SC, Parham R, Freemantle N, Forbes A, Cooper V. Understanding patients' adherence-related beliefs about medicines prescribed for long-term conditions: a meta-analytic review of the Necessity-Concerns Framework. PLoS One. 2013;8(12):e80633.

9. Hugon A, Roustit M, Lehmann A, et al. Influence of intention to adhere, beliefs and satisfaction about medicines on adherence in solid organ transplant recipients. Transplantation. 2014;98(2):222-228.

10. Ladova K, Matoulkova P, Zadak Z, et al. Self-reported adherence by MARS-CZ reflects LDL cholesterol goal achievement among statin users: validation study in the Czech Republic. J Eval Clin Pract. 2014; 20(5):671-677.

11. Matoulkova P, Krulichova IS, Macek K, et al. Chronically ill Czech patients' beliefs about medicines: the psychometric properties and factor structure of the BMQ-CZ. Ther Innov Regul Sci. 2013;47(3): 341-348.

12. Horne R, Weinman J, Hankins M. The beliefs about medicines questionnaire: the development and evaluation of a new method for assessing the cognitive representation of medication. Psychol Health. 1999;14(1):1-24.

13. Horne R, Weinman J. Self-regulation and self-management in asthma: exploring the role of illness perceptions and treatment beliefs in explaining non-adherence to preventer medication. Psychol Health. 2002; 17(1):17-32.

14. Cohen J. Statistical Power Analysis for the Behavioral Sciences. 2nd ed. New York: Lawrence Erlbaum Associates; 1988.

15. Denhaerynck K, Dobbels F, Cleemput I, et al. Prevalence, consequences, and determinants of nonadherence in adult renal transplant patients: a literature review. Transpl Int. 2005;18(10):1121-1133.

16. Dedinska I, Miklusica J, Osinova D, Vojtko M, Galajda P, Mokan M. Kidney transplantation and non-adherence. Aktuality $v$ nefrologii. 2016; 22(4):126-129.

17. Denhaerynck K, Steiger J, Bock A, et al. Prevalence and risk factors of non-adherence with immunosuppressive medication in kidney transplant patients. Am J Transplant. 2007;7(1):108-116.

18. Butler JA, Peveler RC, Roderick P, Horne R, Mason JC. Measuring compliance with drug regimens after renal transplantation: comparison of self-report and clinician rating with electronic monitoring Transplantation. 2004;77(5):786-789.
19. Griva K, Davenport A, Harrison M, Newman SP. Non-adherence to immunosuppressive medications in kidney transplantation: intent vs forgetfulness and clinical markers of medication intake. Ann Behav Med. 2012;44(1):85-93

20. Massey EK, Tielen M, Laging M, et al. The role of goal cognitions, illness perceptions and treatment beliefs in self-reported adherence after kidney transplantation: a cohort study. J Psychosom Res. 2013;75(3): 229-234.

21. Schmid-Mohler G, Thut MP, Wüthrich RP, Denhaerynck K, de Geest S. Non-adherence to immunosuppressive medication in renal transplant recipients within the scope of the Integrative Model of Behavioral Prediction: a cross-sectional study. Clin Transplant. 2010;24(2):213-222.

22. Chisholm-Burns M, Pinsky B, Parker G, et al. Factors related to immunosuppressant medication adherence in renal transplant recipients. Clin Transplant. 2012;26(5):706-713.

23. Kak K, Burger C, Treleaven D, Wallace C. Patient self-reported adherence to and perceptions towards immunosuppressive medications at the Outpatient Renal Transplant Clinic at St. Joseph's Healthcare Hamilton (SJHH) [abstract]. Am J Transplant. 2015;15.

24. Nevins TE, Nickerson PW, Dew MA. Understanding medication nonadherence after kidney transplant. J Am Soc Nephrol. 2017;28(8) 2290-2301.

25. Tielen M, van Staa AL, Jedeloo S, van Exel NJ, Weimar W. Q-methodology to identify young adult renal transplant recipients at risk for nonadherence. Transplantation. 2008;85(5):700-706.

26. Jamieson NJ, Hanson CS, Josephson MA, et al. Motivations, challenges, and attitudes to self-management in kidney transplant recipients: a systematic review of qualitative studies. Am J Kidney Dis. 2016;67(3): 461-478.

27. De Geest S, Burkhalter H, Bogert L, et al. Describing the evolution of medication nonadherence from pretransplant until 3 years posttransplant and determining pretransplant medication nonadherence as risk factor for post-transplant nonadherence to immunosuppressives: the Swiss Transplant Cohort Study. Transpl Int. 2014;27(7):657-666.

28. Muduma G, Shupo FC, Dam S, et al. Patient survey to identify reasons for non-adherence and elicitation of quality of life concepts associated with immunosuppressant therapy in kidney transplant recipients. Patient Prefer Adherence. 2016;10:27-36.

29. Gadkari AS, McHorney CA. Unintentional non-adherence to chronic prescription medications: how unintentional is it really? BMC Health Serv Res. 2012;12:98.

30. Vytrisalova M, Touskova T, Ladova K, et al. Adherence to oral bisphosphonates: 30 more minutes in dosing instructions matter. Climacteric. 2015;18(4):608-616.

31. Tielen M, van Exel J, Laging M, et al. Attitudes to medication after kidney transplantation and their association with medication adherence and graft survival: a 2-year follow-up study. J Transplant. 2014;2014: 675301.

32. Low JK, Williams A, Manias E, Crawford K. Interventions to improve medication adherence in adult kidney transplant recipients: a systematic review. Nephrol Dial Transplant. 2015;30(5):752-761.

33. Chisholm-Burns MA, Spivey CA, Tolley EA, Kaplan EK. Medication therapy management and adherence among US renal transplant recipients. Patient Prefer Adherence. 2016;10:703-709.
Patient Preference and Adherence

\section{Publish your work in this journal}

Patient Preference and Adherence is an international, peer-reviewed, open access journal that focuses on the growing importance of patient preference and adherence throughout the therapeutic continuum. Patient satisfaction, acceptability, quality of life, compliance, persistence and their role in developing new therapeutic modalities and compounds to optimize

\section{Dovepress}

clinical outcomes for existing disease states are major areas of interest for the journal. This journal has been accepted for indexing on PubMed Central. The manuscript management system is completely online and includes a very quick and fair peer-review system, which is all easy to use. Visit http://www. dovepress.com/testimonials.php to read real quotes from published authors. 